\title{
The Exosporium of Clostridium pasteurianum
}

\author{
By B. M. MACKEY AND J. G. MORRIS \\ Department of Botany and Microbiology, School of Biological Sciences, \\ The University College of Wales, Aberystwyth, SY23 3 DA
}

(Received 2 I June I972; revised I4 July 1972)

\begin{abstract}
SUMMARY
Autolytically liberated spores of Clostridium pasteurianum possessed an openended exosporium, which scanning electron microscopy showed as a flimsy integument draped around the rigid spore body. Critical point drying prevented collapse of the exosporium and a material connexion between spore and exosporium was observed in specimens prepared by this technique. A variety of electron-microscopic techniques showed that the exosporium possessed a relatively simple multilamellar structure, each lamella consisting of subunits arranged in paracrystalline array which in surface view displayed hexagonal symmetry. Possible model structures based on these findings are considered.
\end{abstract}

\section{INTRODUCTION}

The spores of several species of Bacillus and Clostridium are contained in sac-like exosporia. These may be tightly or loosely fitting, quite simple in structure or complexly compounded of several distinct layers. Most is known of the loose-fitting exosporium of Bacillus cereus which consists of an outer layer bearing a nap of hair-like projections ( $25 \mathrm{~nm}$ deep) separated by an intermediary layer $(6 \mathrm{~nm})$ from a basal layer $(19 \mathrm{~nm})$ which appears quad rilamellar in cross-section, and as a hexagonally ordered lattice of subunits in surface view (Gerhardt \& Ribi, 1964). Homogenous fragments of this exosporium contained protein $(52 \%)$, amino and neutral polysaccharides $(20 \%)$ and lipids (18\%) (Matz, Beaman \& Gerhardt, 1970); such fragments solubilized by treatment with sodium dodecyl sulphate could re-aggregate to yield paracrystalline sheets with the same hexagonal lattice symmetry as the native exosporium (Beaman, Pankratz \& Gerhardt, I97I). A similar 'typical' exosporium has been found on spores of some strains of B. megaterium (Beaman, Pankratz \& Gerhardt, 1972), but, although some species of Clostridium possess this form of exosporium (Hoeniger \& Headley, I969; Samsonoff, Hashimoto \& Conti, I970), three types of exosporium have been identified in Clostridium strains (Hodgkiss, Ordal \& Cann, I967) and even closely related species of Clostridium produce exosporia of quite different structure (e.g. the butyric clostridia examined by Rousseau, Hermier \& Bergere, I971).

Mackey \& Morris (1971) obtained some evidence that the exosporium of Clostridium pasteurianum was open at one end, but were uncertain whether this was due to rupture during specimen preparation. This paper confirms that the exosporium of $C$. pasteurianum is indeed open-ended and describes ultrastructural details revealed by a variety of electron microscopic procedures. 


\section{METHODS}

Organism. Clostridium pasteurianum (strain w-5) ATCC 60I3 kindly supplied by Mrs Winifred Ego, University of Hawaii, Honolulu, was grown anaerobically and maintained in the sporulated state on slopes of defined growth medium (Mackey \& Morris, 1971).

Preparation of a washed spore suspension. Freshly prepared glucose, ammonium salts plus vitamins medium (500 ml) with calcium carbonate (Mackey \& Morris, I97I) was inoculated with heat-shocked spore suspension $(2 \mathrm{ml})$ and incubated anaerobically at $35^{\circ} \mathrm{C}$ until maximum sporulation had occurred. The culture was then maintained at $37{ }^{\circ} \mathrm{C}$ for 2 to 3 weeks, during which time spores were autolytically released. The rate of autolysis varied from culture to culture and only those preparations which consisted almost entirely of free spores were taken for electron microscopy.

The spore suspension was decanted from the compacted residue of calcium carbonate, and harvested by centrifuging at $17000 \mathrm{~g}$ for $20 \mathrm{~min}$ at $2{ }^{\circ} \mathrm{C}$. The spores were washed and resuspended in sterile water to a density of approx. $10^{8} / \mathrm{ml}$.

\section{Electron microscopy}

Preparation of stained ultrathin sections. The method used was essentially that described by Mackey \& Morris (197I) save that the ethanol-dehydrated specimen cubes were transferred to propylene oxide prior to embedding in Araldite.

Negative staining. An aqueous suspension of spores was centrifuged at $2000 \mathrm{~g}$ for $10 \mathrm{~min}$ and the pellet resuspended in $2 \%(\mathrm{w} / \mathrm{v})$ aqueous uranyl acetate. Droplets of this suspension, removed immediately or after $2 \mathrm{~h}$, were transferred to formvar-coated 200-mesh copper grids and excess liquid drawn off with filter paper.

Freeze etching. The procedure has been described by Short \& Walker (1971).

Preparation of platinum-shadowed specimens. Celloidin-coated 200-mesh copper grids were attached by dual-surfaced adhesive tape to a clean microscope slide. Using an atomizer, an aqueous suspension of spores was sprayed on to the grids which were then dried in air, shadowed with platinum (at an angle of approx. $20^{\circ}$ ) and finally coated with a very light film of carbon using an NGN coating unit (NGN Ltd, Accrington, Lancashire).

Preparation of carbon replicas. Spores mounted on formvar-coated 200-mesh copper grids were platinum shadowed and carbon coated (as above); carbon replicas were then made by the method of Bradley \& Williams (1957).

Critical-point drying. One drop of the aqueous spore suspension was placed on a formvarcoated 200-mesh copper grid. When sufficient time had elapsed to allow the spores to settle onto the grid, excess water was shaken off and the grid was placed vertically in a grid-holder to facilitate handling as the unfixed spores were dehydrated by serial passage through $50 \%$, $70 \%$ and $90 \%(\mathrm{v} / \mathrm{v})$ ethanol: water mixtures (30 s in each) and finally, after $30 \mathrm{~s}$ immersion in absolute ethanol, placed in amyl acetate. The specimen was then quickly transferred to an apparatus of the type described by Anderson (195I) in which the amyl acetate was quickly replaced by liquid $\mathrm{CO}_{2}$. The pressure chamber was sealed and the temperature raised to $45^{\circ} \mathrm{C}$, i.e. above the critical point of $\mathrm{CO}_{2}\left(3 \mathrm{r}^{\circ} \mathrm{C}\right)$. The resulting transition from liquid to gaseous $\mathrm{CO}_{2}$ was accomplished without any passage of a liquid/gas boundary through the specimen so that when the $\mathrm{CO}_{2}$ was allowed to escape, the dry specimen which remained was free from much of the distortion (collapse) caused by surface tension forces during the more usual air-drying procedures.

Optical diffractometric analysis. A method of determining periodicities in electron micrographs using optical transforms was described by Klug \& Berger (I964); our optical 

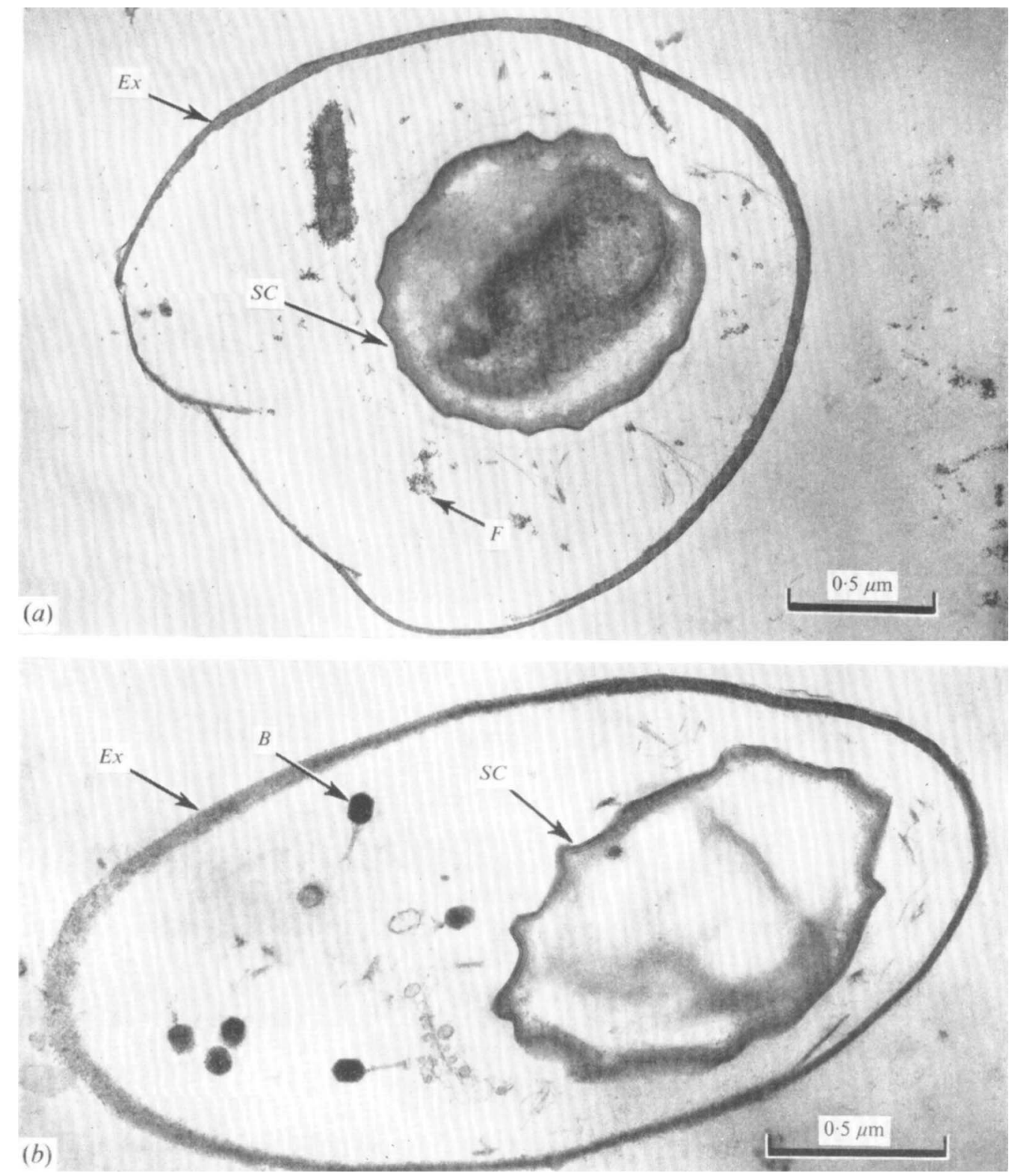

Fig. I. (a) Entrapment of ferritin molecules in the perisporal space. Ex, Exosporium; $F$, ferritin; $S C$, spore coat. (b) Entrapment of $\mathrm{T} 6$ coliphage particles in the perisporal space. $B$, Bacteriophage.

transforms were obtained from negative electron-microscope plates using a prototype diffractometer being developed by Rank Precision Industries Ltd, Leicester.

Scanning electron microscopy. Aluminium-coated spores were examined in a Cambridge 'Stereoscan' electron microscope (Cambridge Scientific Instruments Ltd). 

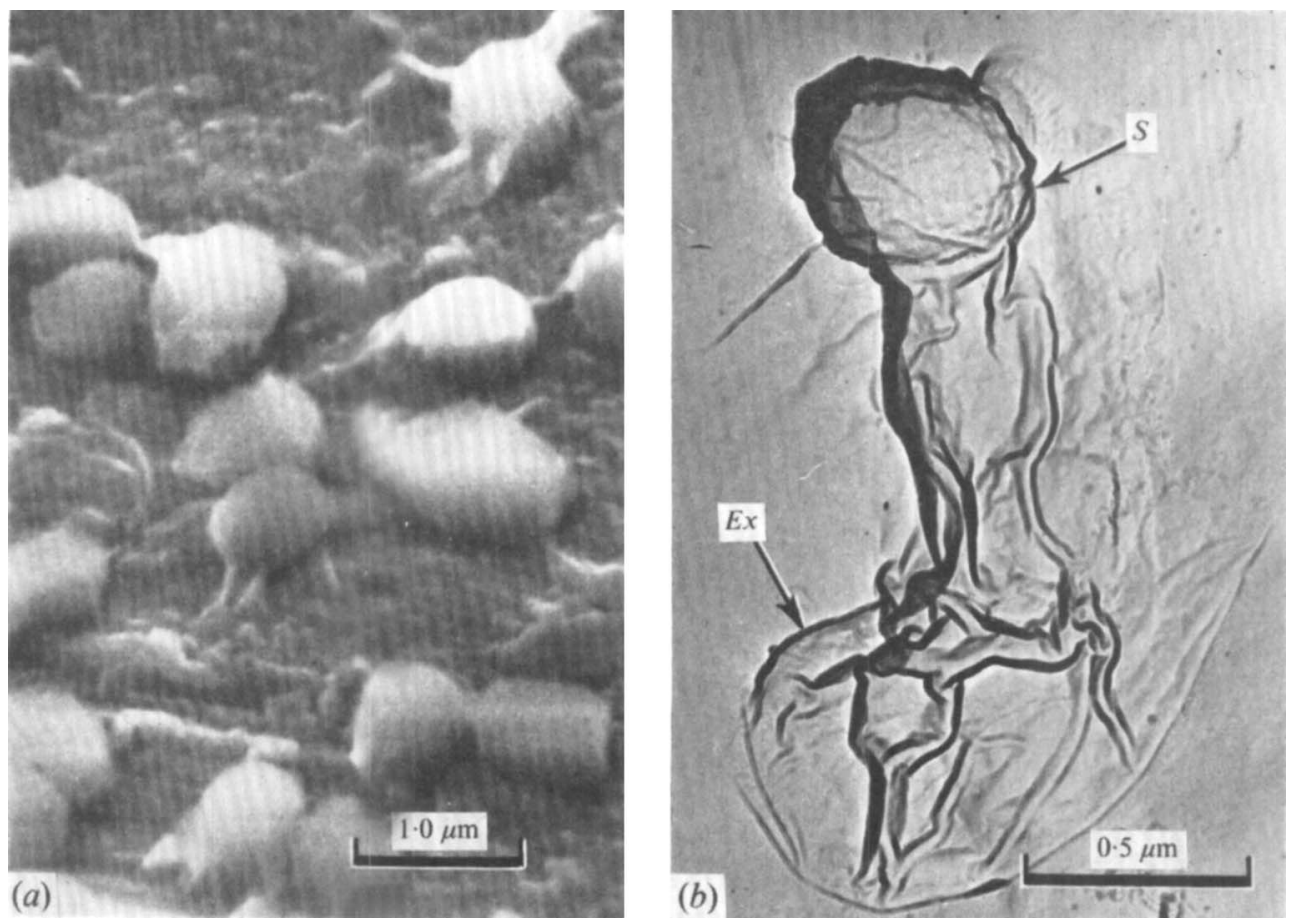

Fig. 2. (a) Scanning electron micrograph of spores of Clostridium pasteurianum, showing flimsy exosporium draped around spore body. (b) Carbon replica of spore $(S)$ with collapsed exosporium $(E x)$.

\section{RESULTS}

Presence of an opening in the exosporium. Mackey \& Morris (1971) found that the exosporium of Clostridium pasteurianum apparently contained a basal hole visible in medial longitudinal sections. To confirm the presence of this opening in the untreated exosporium, spore suspensions were mixed with electron-dense particles (ferritin, I I nm diameter; T6 coliphage, $65 \mathrm{~nm}$ width) to see whether the particles entered the space between the spore and exosporium.

Ferritin. A mixed aqueous suspension $(2 \mathrm{ml})$ of $3.2 \times 10^{8}$ spores with $2.5 \mathrm{mg}$ of ferritin was slowly shaken in a $25 \mathrm{ml}$ conical flask for $\mathrm{I} \cdot 5 \mathrm{~h}$ at room temperature. Two $0.5 \mathrm{ml}$ samples were removed and centrifuged at $2000 \mathrm{~g}$ for $5 \mathrm{~min}$ at $2{ }^{\circ} \mathrm{C}$. One pellet was then suspended in the fixative of Kellenberger, Ryter \& Sechaud (1958), while the other pellet was washed by resuspending in distilled water and recentrifuging. Sections (see Methods) of spores from both pellets showed the presence of large numbers of ferritin molecules in the space enclosed by the exosporium. The single wash almost entirely removed extraneous ferritin but ferritin molecules remained trapped within the perisporal space (Fig. I $a$ ).

$T 6$ coliphage. An aqueous suspension ( $\mathrm{I} \mathrm{ml}$ ) containing $10^{8}$ spores and $2 \cdot 7 \times 10^{11} \mathrm{~T} 6$ phage particles was incubated with gentle shaking for $2.5 \mathrm{~h}$ at room temperature and then divided into two portions. One was centrifuged at $2000 \mathrm{~g}$ for $5 \mathrm{~min}$, the supernatant discarded and the pellet resuspended in Kellenberger et al. (1958) fixative. The other portion was similarly centrifuged but the pellet was washed with $2 \mathrm{ml}$ of water before being recentrifuged and suspended in fixative. Again sections showed (Fig. I $b$ ) that the particles (easily identifiable by their characteristic shape) had gained access to the perisporal space and had not been expelled by the washing procedure. 


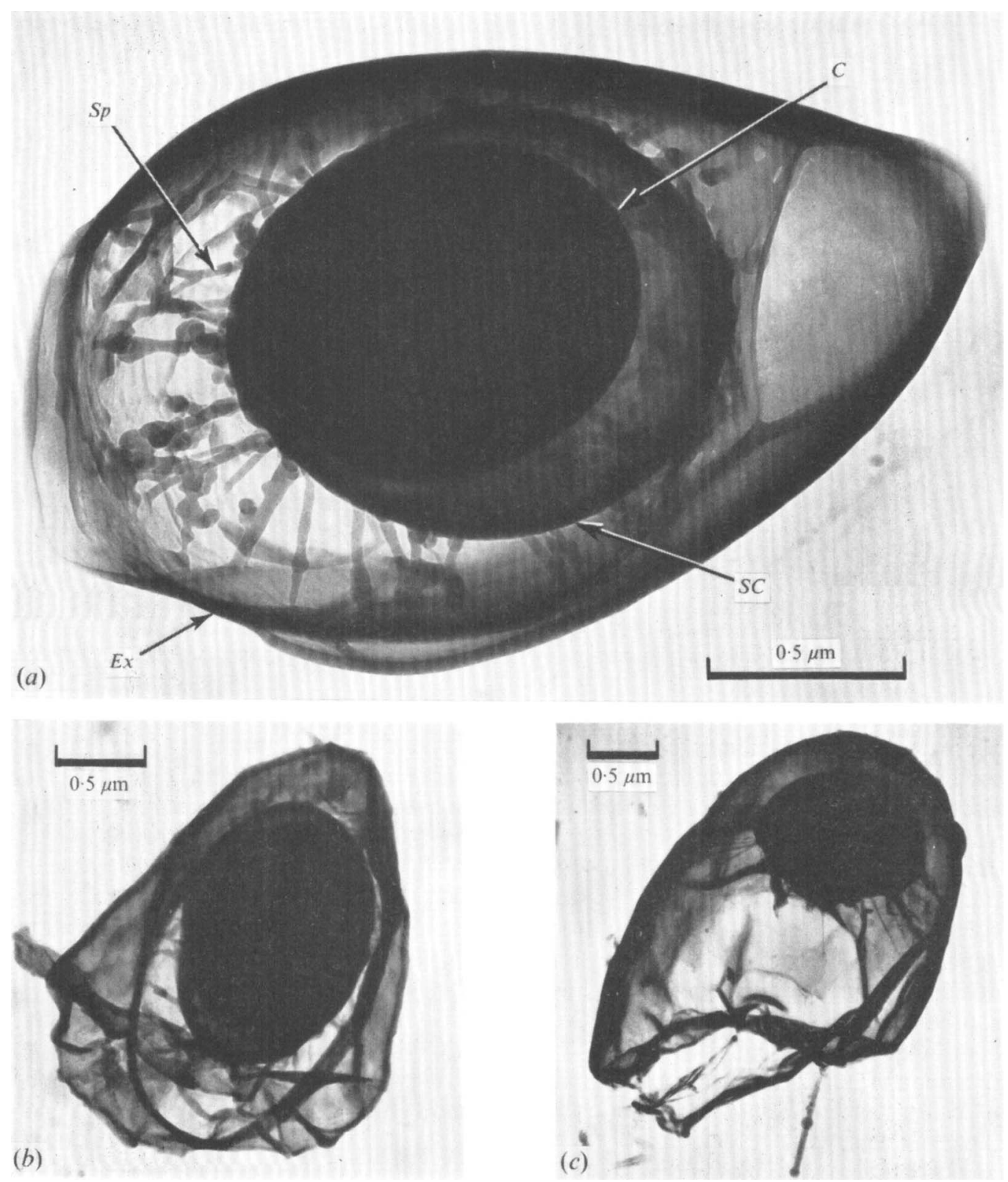

Fig. 3. Critical-point-dried preparations of autolytically liberated spores of Clostridium pasteurianum. (a) The spore, consisting of an electron-dense spore core $(C)$ in its more electron-lucent coat $(S C)$, seems to be slung by 'suspensors' $(S p)$ within its exosporium $(E x)$. $(b)$ View of the spore seen through the open base of the exosporium. (c) Side view of the spore within its exosporium with a partially detached 'suspensor'. 


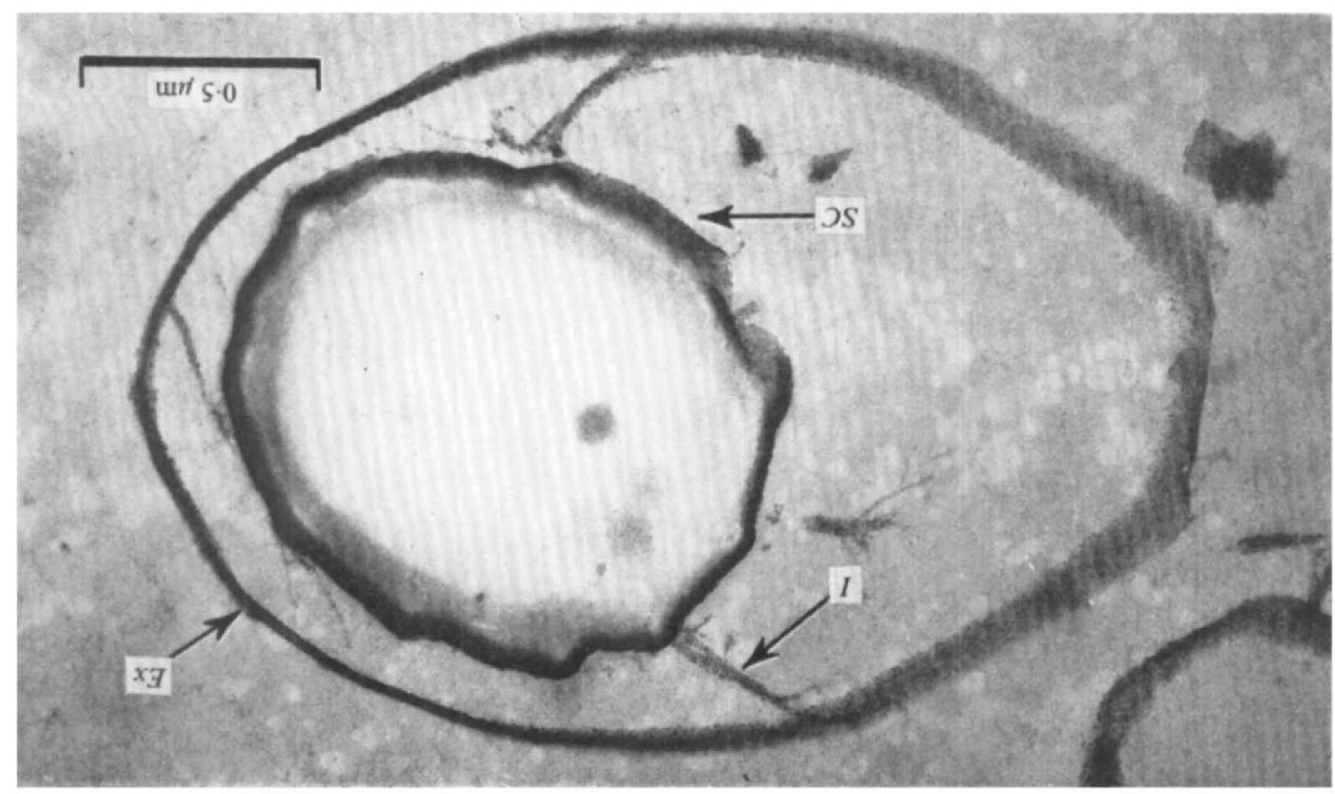

Fig. 4. Longitudinal section of complete spore of Clostridium pasteurianum showing inclusions $(I)$ bridging the perisporal gap between the exosporium $(E x)$ and the spore coat $(S C)$.

Relationship between the spore and its exosporium. Scanning electron micrographs showed the exosporium as a flimsy integument draped around the rigid spore body (Fig. $2 a$ ). Similar collapse of the exosporium during the preparation of carbon replicas (Fig. $2 b$ ) gave rise to obviously distorted preparations quite unlike the characteristically shaped exosporium seen in thin sections (Mackey \& Morris, I97I). In an attempt to minimize distortion and to retain the 'normal' three-dimensional structure of the exosporium we used the criticalpoint drying method of specimen preparation (Methods). Fig. 3(a) shows the dense spore core within its spore coat apparently slung by 'suspensors' from the exosporium. We interpret Fig. $3(b)$ as a view of the spore seen through the open base of the exosporium, this opening being even more obvious in Fig. $3(c)$ in which surface ridges (folds) of the exosporium are also quite plainly seen. We had previously obtained sections in which the occasional inclusion could be discerned apparently connecting the exosporium with the spore coat (Fig. 4). In a freeze-etched, fractured specimen (Fig. $5 b$ ) there was some indication of the existence of a loosely woven layer interposed between the broken exosporium and the spore coat.

\section{Fine structure of the exosporium}

Transverse sections of the exosporium of Clostridium pasteurianum (Fig. $8 a$; Mackey \& Morris, I97I) showed that it was a simple, multilamellar structure which was thickest at its apex and thinnest towards its base due to some of the lamellae having extended less far than others. The interlamellar repeat distance (centre-to-centre spacing) was about $5 \mathrm{~nm}$ and the repeat distance (centre to centre) of component subunits in each lamella was again about $5 \mathrm{~nm}$ (and not 2.I $\mathrm{nm}$ as previously reported in Mackey \& Morris, 1971). The outermost lamella resembled those internal to it and no covering membrane or nap was dis- 

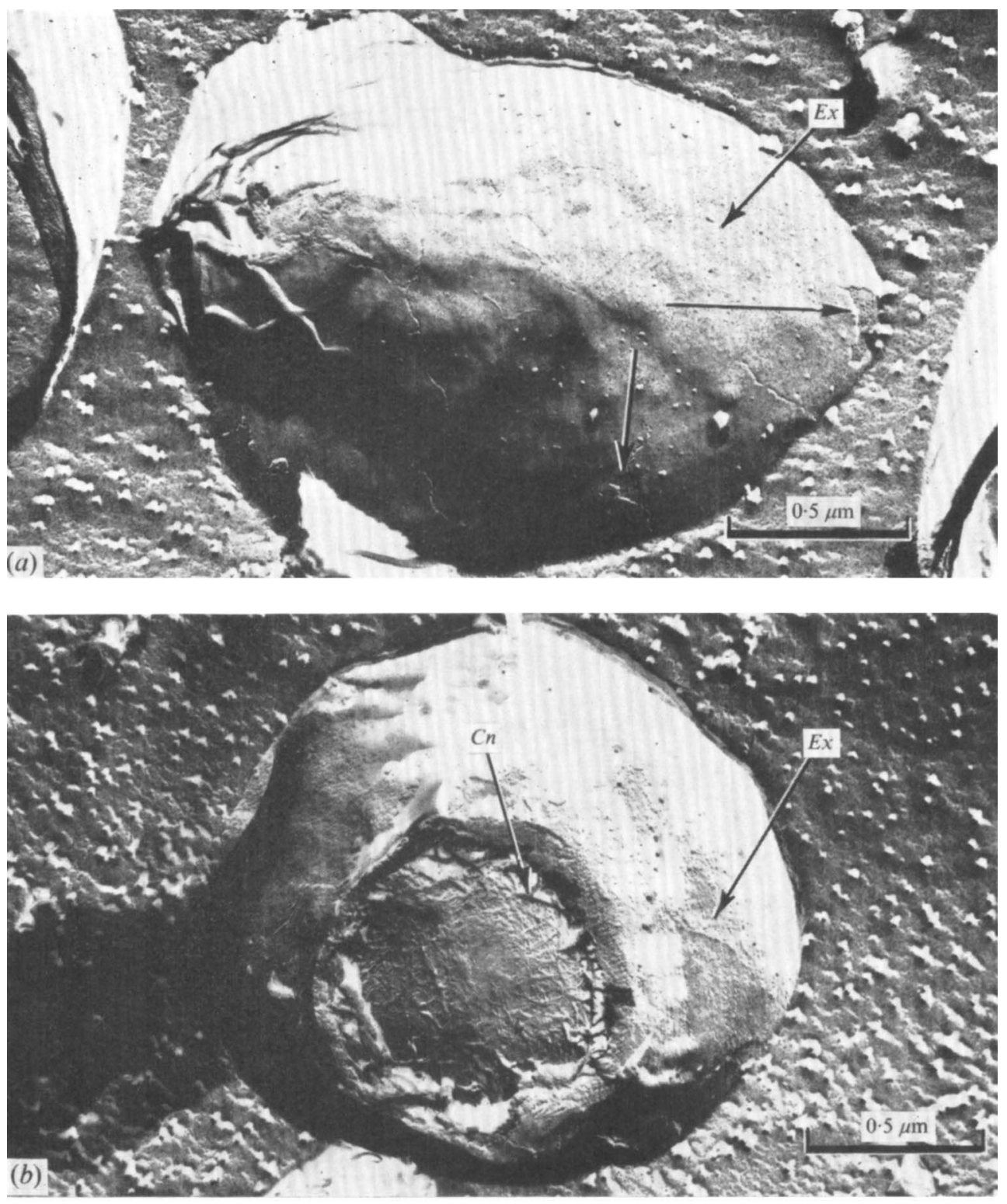

Fig. 5. Freeze-etched specimens of Clostridium pasteurianum spores (prepared and photographed by Mr J. Short and Dr P. D. Walker). (a) Complete spore showing finely ordered outer surface of the exosporium $(E x)$ with subsurface layer(s) visible through the occasional rent (arrowed) in this surface. $(b)$ Fractured specimen showing possible presence of connective material $(C n)$ between spore coat and exosporium $(E x)$.

cernible. In a freeze-etched preparation of a complete spore (Fig. $5 a$ ) the surface of the exosporium was very finely ordered; only where minute breaks had occurred was a more pronounced pattern of more widely separated pits discernible in deeper layers. Negative staining (Fig. 6) produced evidence of a lattice structure which in surface view had apparently hexagonal periodicity; in some lamellae the foci of staining were spaced $5 \mathrm{~nm}$ apart 

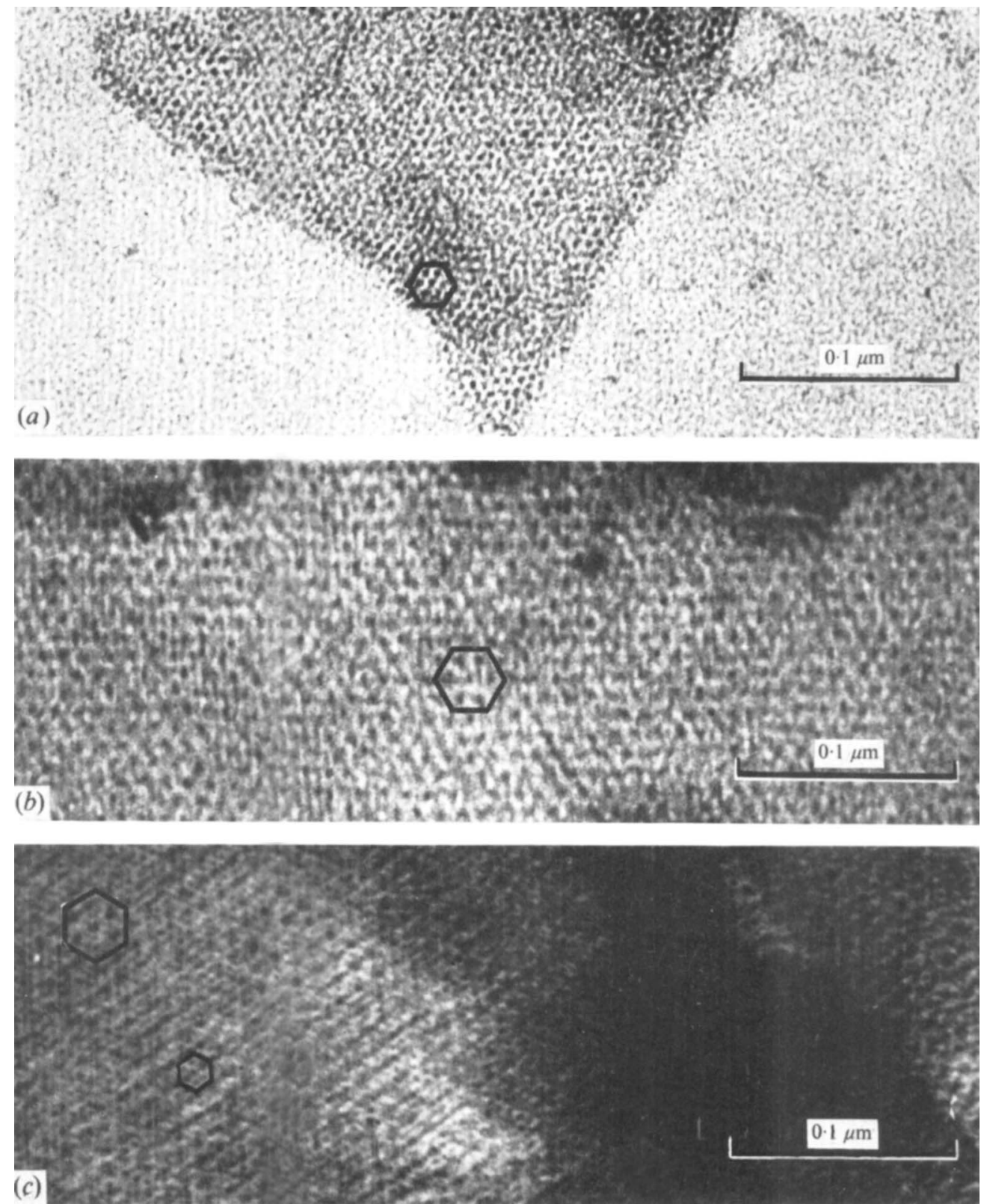

Fig. 6. Negatively stained fragments of exosporium of Clostridium pasteurianum. (a) Thin flake displaying $5 \mathrm{~nm}$ hexagonal periodicity. (b) Specimen displaying predominantly $10 \mathrm{~nm}$ hexagonal periodicity. (c) Specimen displaying both $5 \mathrm{~nm}$ and $10 \mathrm{~nm}$ hexagonal periodicities. 

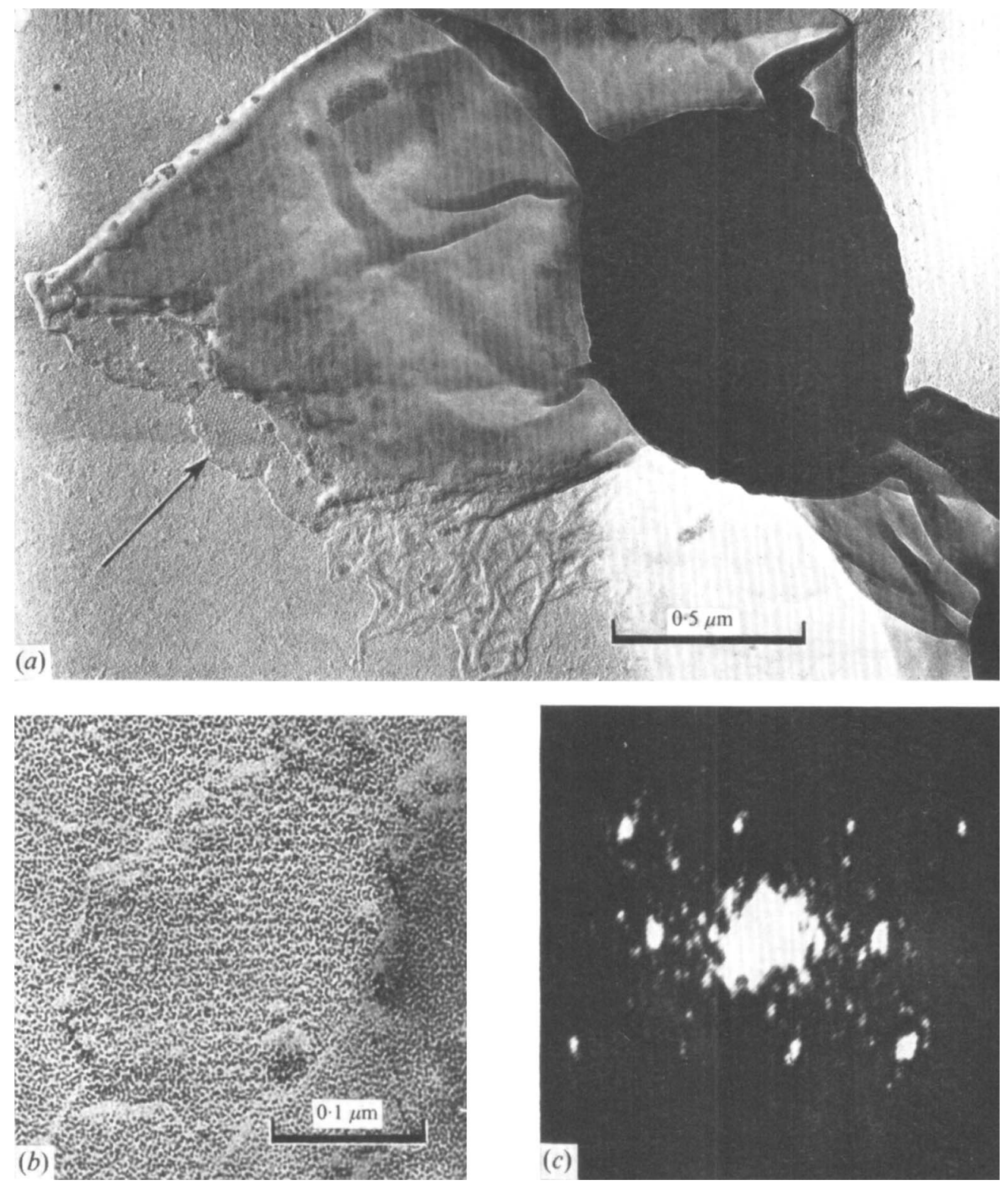

Fig. 7. (a) Platinum-shadowed spore of Clostridium pasteurianum showing outer and inner surfaces of the exosporium. The substructure of the inner surface (arrowed) is most pronounced. $(b)$ Enlarged view of the segment of the platinum-shadowed inner surface of the exosporium arrowed in $(a)$. (c) Optical diffraction pattern given by $(b)$.

(Fig. 6a), in other preparations the repeat distance was Io $\mathrm{nm}$ (Fig. 6b), while in others both $5 \mathrm{~nm}$ and $\mathrm{I} 0 \mathrm{~nm}$ periodicities were displayed (Fig. $6 c$ ). In platinum-shadowed specimens (Fig. $7 a)$ the subunits were much more prominent on the inner surface of the exosporium than on its outer surface. Fig. $7(c)$ shows the optical diffraction pattern given by a much magnified segment of the inner surface of the platinum-shadowed exosporium (Fig. $7 b$ ). Analysis of 


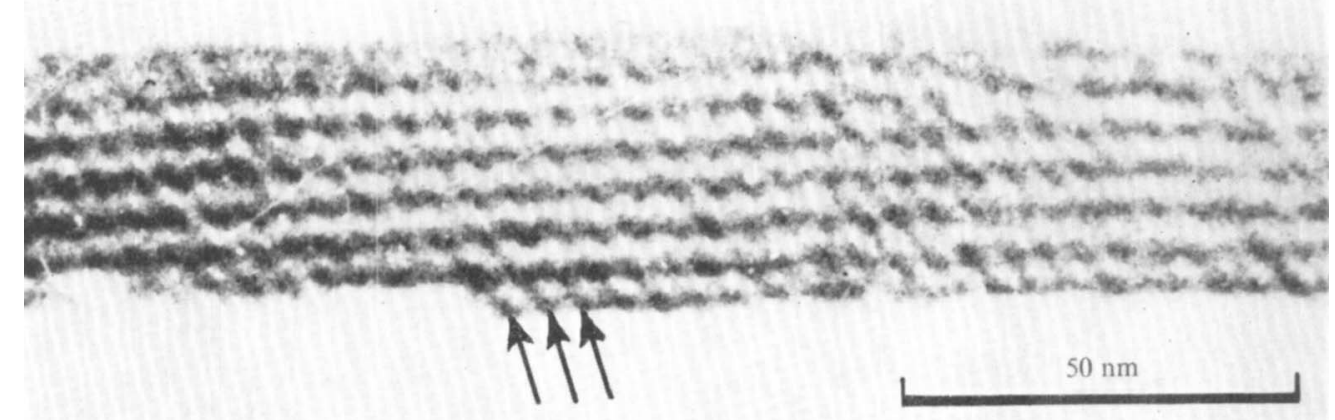

(a)

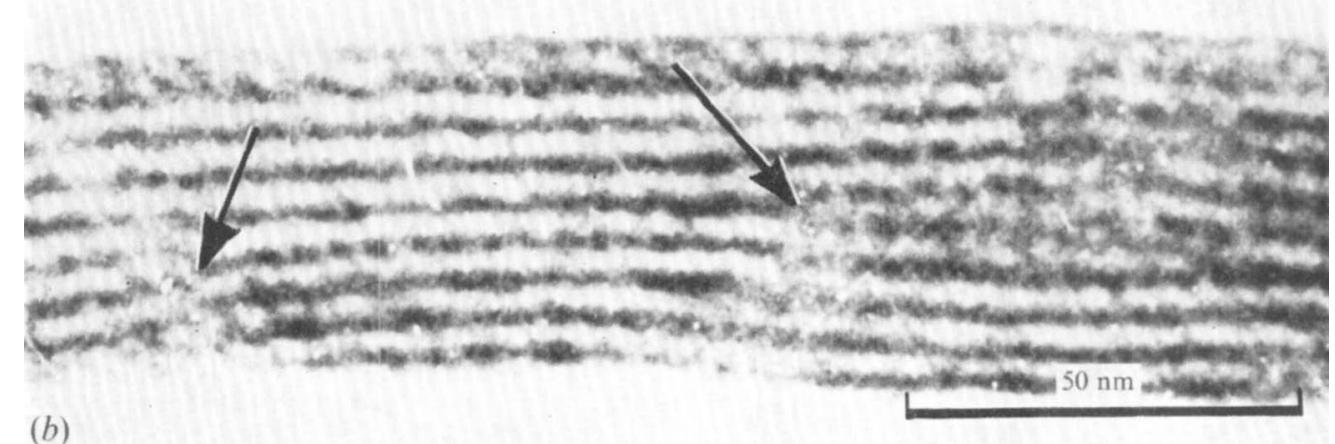

(b)
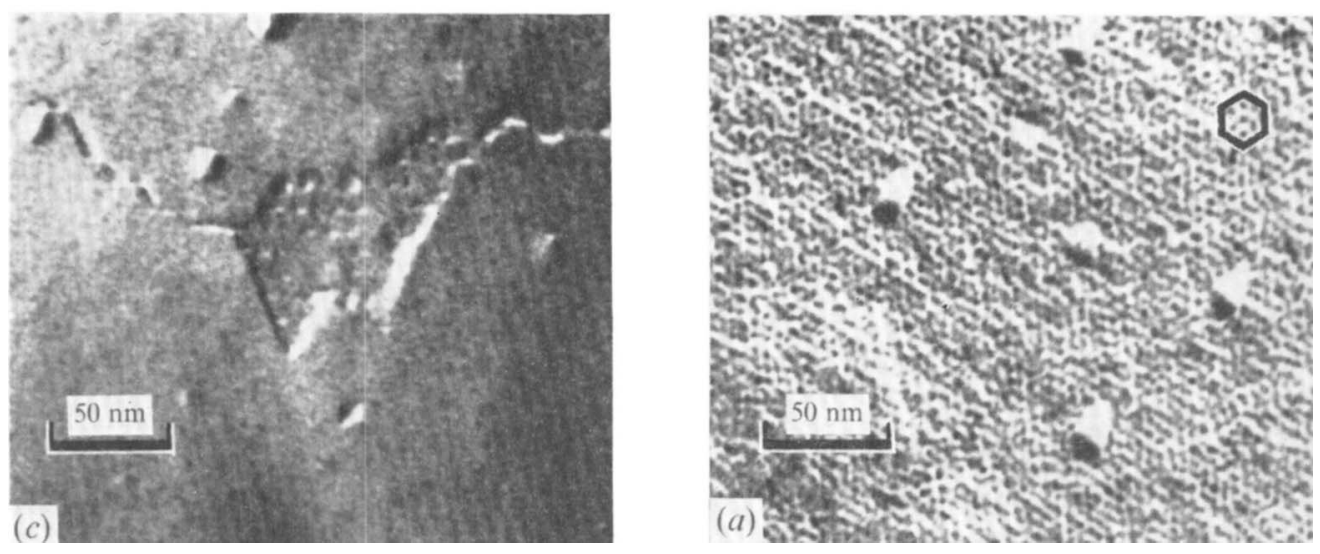

Fig. 8. (a) Lead citrate stained transverse section of the exosporium of Clostridium pasteurianum showing lamellae composed of subunits (arrowed); the subunits are regularly arranged with a centre to centre repeat distance of approx. $5 \mathrm{~nm}$. (b) Transverse section of exosporium showing probable sessile edge dislocations of large Burgers vector (arrowed). (c) Enlarged portion of freezeetched specimen shown in Fig. 5(a), showing hexagonal pattern of pits (IO nm periodicity) in infralayer revealed by damage to surface of exosporium. $(d)$ Enlarged portion of freeze-etched specimen shown in Fig. 5(a), showing hexagonal array of subunits (with $5 \mathrm{~nm}$ periodicity) forming the outer surface of the exosporium. 


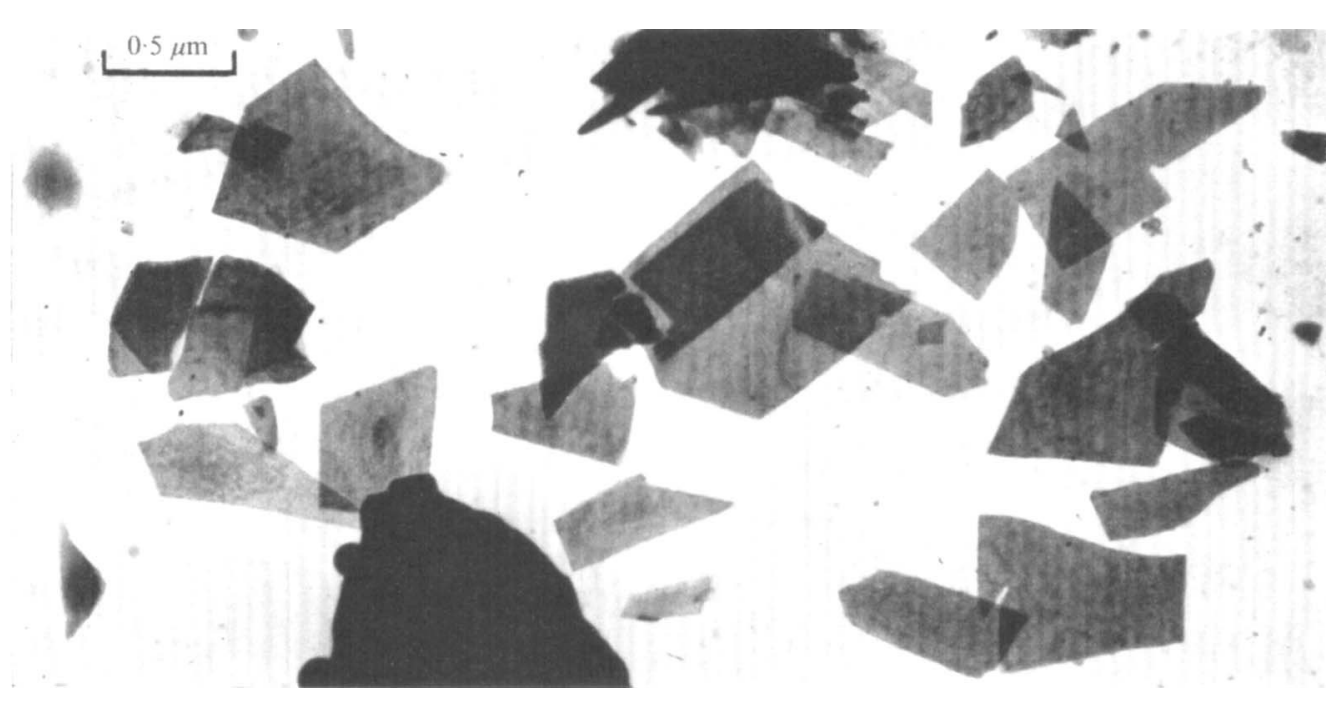

Fig. 9. Fragments of exosporium of Clostridium pasteurianum.

the angles and periodicities in this pattern confirmed the $5 \mathrm{~nm}$ separation and hexagonal arrangement of the subunits, reinforcing the view of the lattice structure obtained by direct visual examination both of negatively stained preparations such as that shown in Fig. $6(a)$, and of transverse sections (Fig. $8 a, b$ ). Further evidence of the paracrystalline nature of the structure was provided by the distinctive appearance of fragments of disintegrated exosporium (Fig. 9).

\section{DISCUSSION}

The accessibility of the perisporal space to ferritin and phage particles confirmed our previous finding that the exosporium of Clostridium pasteurianum only incompletely envelopes the spore and poses no permeability barrier. The terminal opening can be of considerable size (Fig. $3 b, c$ ) and, although fraying and other damage during centrifuging and subsequent manipulations could enlarge this hole, all the evidence suggests that the exosporium of autolytically liberated spores of this strain is invariably open-ended. No spore appendages were observed and one is left to speculate whether the exosporial opening serves any useful function. After germination, the outgrowing vegetative cell invariably emerges through this opening leaving behind a seemingly intact exosporium (Mackey \& Morris, I972).

During the synthesis of exosporium the perisporal space is filled with 'mother cell' cytoplasm, and, although this is perhaps the first portion of the maternal plasm to undergo autolysis (Mackey \& Morris, I97I), we do not know how far this lysis proceeds. Thus the perisporal space may (i) be filled with residual maternal cytoplasm (albeit partially lysed), (ii) contain some novel connective plasm, (iii) be traversed by filamentous 'suspensors' whereby the spore is slung within the exosporial sac, or (iv) be empty of plasm or connective strands. In any event, because of the basal opening in the exosporium the perisporal contents will be in direct contact with the suspending medium.

The micrographs obtained after critical point drying (Fig. 3) suggest the existence of 'suspensors'. These are easily distinguished from folds in the exosporium (Fig. $3 c$ ) and are particularly evident in Fig. 3(a). The appearance of the electron-dense spore core within its 

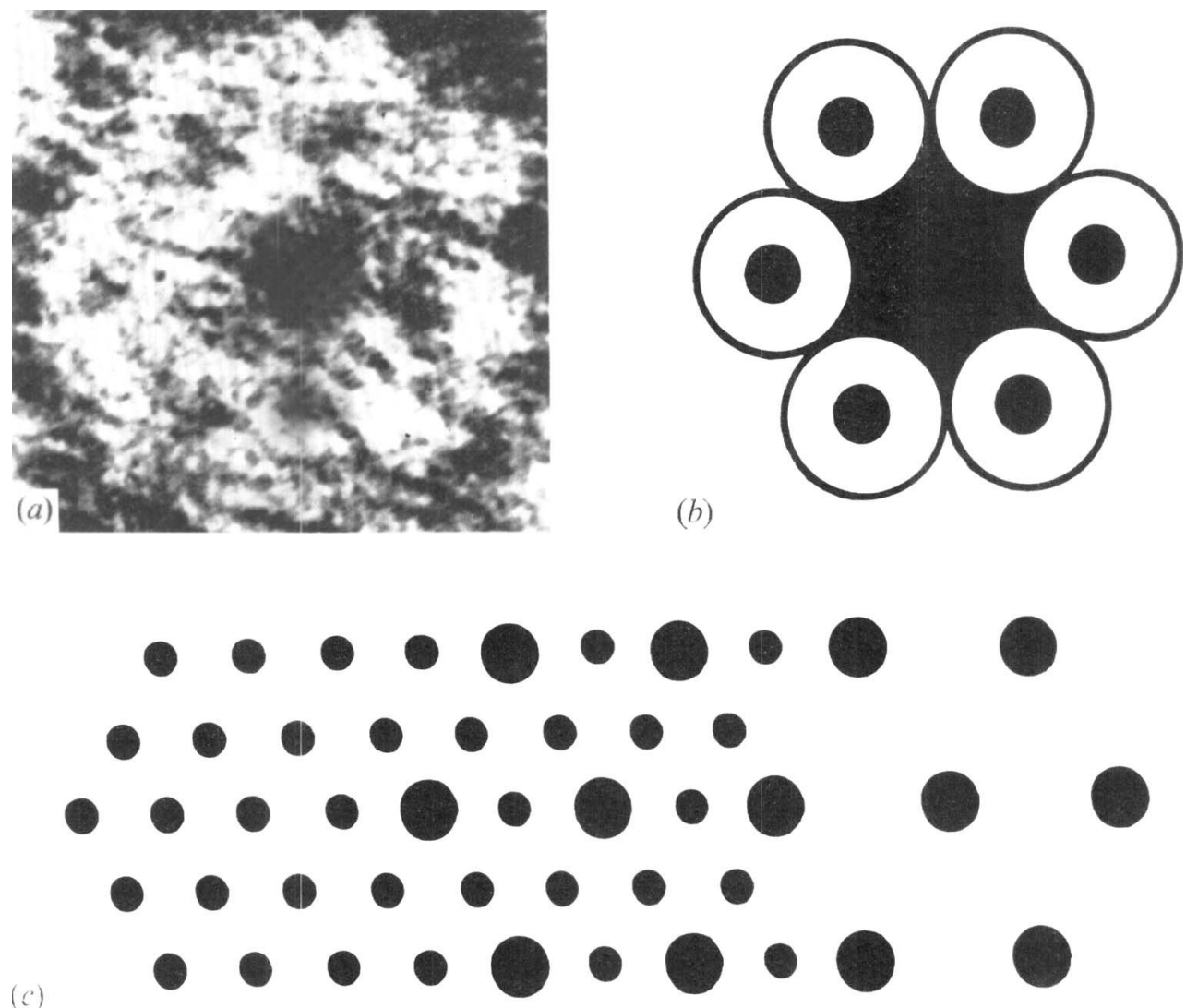

Fig. I0. (a) Much enlarged detail of the negatively stained exosporium of Clostridium pasteurianum shown in Fig. 6(c) (magnified approximately $\times 3000000)$. (b) Plausible interpretation of $(a)$; a model in which doughnut-shaped subunits are arranged in hexagonal array. $(c)$ Model demonstrating how the dual $5 \mathrm{~nm}$ and $10 \mathrm{~nm}$ hexagonal periodicities discernible in a negatively stained fragment of exosporium (Fig. $6 c$ ) could result from the superposition of distinctively patterned layers.

more electron-lucent coat (Fig. $3 a$ ) is similar to that of viable spores of Bacillus subtilis viewed by high-voltage electron microscopy (Dupouy, I968), but the connective threads seen in Fig. 3 could still be artifacts produced during sample preparation. In particular they could be denatured threads of material extracted from the spore coat and/or exosporium by the ethanol and amyl acetate treatment that preceded the critical-point drying. Besides the presence of perisporal inclusions in stained sections of spores (Fig. 4) our only evidence for the existence of such connective material is that provided by the freeze-etched, fractured specimen (Fig. $5 b$ ) which seems to indicate the presence of a loosely woven layer between spore coat and exosporium.

The simple multilamellar nature of the exosporium and the regularly $5 \mathrm{~nm}$ spaced subunit structure of each layer was evident in stained, transverse sections (Fig. $8 a$ ). From the manner in which the exosporium was formed within the mother cell plasm, growing from the apex, with the outermost lamellae attaining the greatest length and enclosing progressively shorter inner lamellae (Mackey \& Morris, I97I), it would seem that each lamella may be considered as a paracrystalline sheet whose growth consists of accretion and self-assembly of subunits 
in an extending two-dimensional lattice. In surface views of negatively stained (Fig. 6) and metal-shadowed (Fig. 7) specimens these subunits were arranged in a repeating pattern of regular hexagons, so that overall, in a multilamellar section of exosporium, the subunits are arranged as if in a close-packed hexagonal, three-dimensional lattice. Thus in its fine structure the exosporium of Clostridium pasteurianum might resemble the basal layer of the exosporium of Bacillus cereus (Beaman et al. 197I) and the exosporium of $C$. botulinum type E, OS24AS (Hodgkiss et al. 1967), though with its interunit repeat distance of $5 \mathrm{~nm}$ it even more closely resembles the laminar inclusions found in the perisporal region of Bacillus megaterium MGI9 (Beaman et al. 1972). However, closer examination of the surface views obtained by negative staining and freeze-etching procedures suggests that the exosporial substructure may be more complicated than is indicated by the appearance of transverse sections. Thus, while the freeze-etched specimen (Fig. $5 a, 8 d$ ) demonstrated in the outer surface of the exosporium the expected arrangement of subunits in hexagonal array with $5 \mathrm{~nm}$ periodicity, it also suggested the existence of an infralayer bearing a hexagonally ordered pattern of pits with $10 \mathrm{~nm}$ periodicity (Fig. $8 \mathrm{c}$ ). In the thinnest, negatively stained flakes of exosporium (Fig. $6 a$ ) the foci of staining were hexagonally ordered with $5 \mathrm{~nm}$ periodicity, but in some thicker fragments larger foci of staining predominated, again hexagonally arranged but with Io nm periodicity (Fig. $6 b$ ); both of these patterns were simultaneously discernible in other fragments (Fig. $6 c$, I $a$ ).

It is difficult to reconcile the duality of surface pattern with the proposition that each lamella is composed of identical subunits spaced $5 \mathrm{~nm}$ apart. The model structure shown in Fig. Io $(b)$ supposes the subunits to be 'doughnut' shaped, each with a central hole which forms the smaller focus of negative staining, these being hexagonally disposed around vacant sites which are the larger foci of negative staining. An alternative model shown in Fig. Io $(c)$ supposes that the lamellae only contribute the hexagonally ordered pattern of subunits with $5 \mathrm{~nm}$ periodicity, the interstitial layers (electron-lucent in Fig. $7 a$ ) supplying the hexagonal pattern of $10 \mathrm{~nm}$ periodicity, i.e. of pits in the freeze-etched infralayer (Fig. $8 c$ ) and of larger foci of negative staining (Fig. $6 b$ ).

Neither of these models is entirely convincing, and, until more is known of the composition and shape of subunits liberated from solubilized exosporial fragments, it may not be profitable to indulge in further structural speculations.

We are grateful to Mr J. Short and Dr P. D. Walker of the Wellcome Research Laboratories, Beckenham, for preparing and photographing the freeze-etched specimens shown in Fig. 5, and to Mr J. Jungles of Rank Precision Industries Ltd, Leicester, for assistance with the optical diffractometry. This work was supported by a grant from the Science Research Council.

\section{REFERENCES}

ANDERSON, T. F. (195I). Techniques for the preservation of three-dimensional structure in preparing specimens for the electron microscope. Transactions of the New York Academy of Sciences B 13, I30-134.

Beaman, T. C., Pankratz, H. S. \& Gerhardt, P. (I97I). Paracrystalline sheets reaggregated from solubilized exosporium of Bacillus cereus. Journal of Bacteriology 107, 320-324.

Beaman, T. C., Pankratz, H. S. \& Gerhardt, P. (1972). Ultrastructure of the exosporium and underlying inclusions in spores of Bacillus megaterium strains. Journal of Bacteriology 109, I I98-1 209.

Bradley, D. E. \& Williams, D. J. (1957). An electron microscope study of the spores of some species of the genus Bacillus using carbon replicas. Journal of General Microbiology 17, 75-79.

Dupouy, G. (1968). Electron microscopy at very high voltages. In Advances in Optical and Electron Microscopy, vol. 2, p. 244. Edited by R. Barer and V. E. Coslett. New York \& London: Academic Press. 
GeRHARDT, P. \& RIBI, E. (1964). Ultrastructure of the exosporium enveloping spores of Bacillus cereus. Journal of Bacteriology 88, 1774-1789.

Hodgkiss, W., ORDAL, Z. J. \& CANN, D. C. (1967). The morphology and ultrastructure of the spore and exosporium of some Clostridium species. Journal of General Microbiology 47, 21 3-225.

HoENIGER, J. F. M. \& HeAdley, C. L. (I969). Ultrastructural aspects of spore germination and outgrowth in Clostridium sporogenes. Canadian Journal of Microbiology 15, 106I-1065.

Kellenberger, E., Ryter, A. \& Sechaud, J. (1958). Electron microscopic study of DNA-containing plasms. Vegetative and mature phage DNA as compared with normal bacterial nucleoids in different physiological states. Journal of Biophysical and Biochemical Cytology 4, 671-678.

Klug, A. \& Berger, J. E. (1964). An optical method for the analysis of periodicities in electron micrographs and some observations on the mechanism of negative staining. Journal of Molecular Biology 10, $565-569$.

MACKeY, B. M. \& MoRRIS, J. G. (197I). Ultrastructural changes during sporulation of Clostridium pasteurianum. Journal of General Microbiology 66, I-I 3.

MACKeY, B. M. \& MoRris, J. G. (1972). Calcium dipicolinate-provoked germination, and the outgrowth of spores of Clostridium pasteurianum. Journal of General Microbiology 73, 31 5-324.

Matz, L. L., Beaman, T. C. \& Gerhardt, P. (1970). Chemical composition of exosporium from spores of Bacillus cereus. Journal of Bacteriology ror, 196-201.

Rousseau, M., Hermier, J. \& Bergere, J.-L. (I97I). Structure de certains Clostridium du groupe butyrique. I. Sporulation de Clostridium butyricum et Clostridium saccharobutyricum. Annals d'Institute Pasteur 120, 23-32.

Samsonoff, W. A., Hashimoto, T. \& ConTi, S. F. (1970). Ultrastructural changes associated with germination and outgrowth of an appendage-bearing clostridial spore. Journal of Bacteriology ror, 1038-1045.

SHORT, J. \& WALKER, P. D. (I97I). Structure of bacterial spores as revealed by freeze-etching. In Spore Research 1971 , pp. 189-192. Edited by A. N. Barker, G. W. Gould and J. Wolf. New York and London: Academic Press. 